\title{
Spatiotemporal Control of Amyloid-Like A Plaque Formation Using a Multichannel Organic Electronic Device
}

Erik Gabrielsson, Astrid Armgarth, Per Hammarström, Peter Nilsson and Magnus Berggren

\author{
Linköping University Post Print
}

\section{Tweet}

N.B.: When citing this work, cite the original article.

Original Publication:

Erik Gabrielsson, Astrid Armgarth, Per Hammarström, Peter Nilsson and Magnus Berggren, Spatiotemporal Control of Amyloid-Like A Plaque Formation Using a Multichannel Organic Electronic Device, 2016, Macromolecular materials and engineering (Print), (301), 4, 359-363. http://dx.doi.org/10.1002/mame.201500428

Copyright: Wiley-VCH Verlag

http://www.wiley-vch.de/publish/en/

Postprint available at: Linköping University Electronic Press

http://urn.kb.se/resolve?urn=urn:nbn:se:liu:diva-127750 


\section{WILEY-VCH}

DOI: $10.1002 /(($ please add manuscript number $))$

Article type: Communication

\section{Spatiotemporal Control of Amyloid-Like A $\beta$ Plaque Formation Using a Multi-Channel Organic Electronic Device}

Erik O. Gabrielsson, Astrid Armgarth, Per Hammarström, K. Peter R. Nilsson", and Magnus Berggren*

Dr. E. O. Gabrielsson, A. Armgarth, Prof. M. Berggren

Laboratory of Organic Electronics, Department of Science and Technology, Linköping

University, SE-601 74 Norrköping, Sweden

E-mail: magnus.berggren@liu.se

Prof. P. Hammarström, Dr. K. P. R. Nilsson

IFM-Department of Chemistry, Linköping University, SE-581 83 Linköping, Sweden

E-mail: petni@ifm.liu.se

Keywords: amyloid, fibrils, Alzheimer's disease, bioelectronics, luminescent conjugated oligothiophene, optical probes

Alzheimer's disease ${ }^{[1]}(\mathrm{AD})$ is associated with formation of nanoscopic fibrillar amyloid-beta peptide $(A \beta)$ aggregates which grow to micron sized senile plaques in the brain ${ }^{[2]}$. It is therefore considerable scientific interest in $\mathrm{A} \beta$, and its fundamental properties in a wide sense, in order to understand $\mathrm{AD}$. For the development of new techniques, both for $\mathrm{AD}$ detection and prevention, researchers are dependent on either tissue samples from deceased patients, animal models, or in vitro systems. In vitro systems, such as producing protein aggregates of the A $\beta$ peptide in a test tube by incubation, offers us a simple but rather blunt tool for evaluating aggregation mechanisms or to investigate new detection methods. In addition, microfluidic systems have been used to detect $A \beta$ plaque formation ${ }^{[3]}$, to study $A \beta$ nucleation mechanisms $^{[4]}, A \beta$ toxicity $^{[5]}$, inhibition of $A \beta$ aggregate formation $^{[6,7]}$, and to induce $A \beta$ aggregate dissociation ${ }^{[8]}$. However, the use of microfluidic flows can produce unwanted effects on the aggregation conditions, as it has been shown that the flow rate can change the obtained kinetics ${ }^{[9]}$ and morphology ${ }^{[10]}$ for insulin aggregates. 


\section{WILEY-VCH}

We recently introduced the organic electronic ion pump (OEIP) as a method for creating amyloid-like aggregates at high spatiotemporal resolution as compared to aggregates formed under regular test tube-conditions ${ }^{[11]}$. Combined with a fluorescent probe that is specific for the fibrillar form of misfolded peptides commonly seen in $\mathrm{AD}^{[12]}$, the OEIP allowed us to control and monitor the aggregation of a model peptide system in a highly confined space. The OEIP device is an attractive alternative to microfluidic techniques, as the electrophoretic ion delivery generated by the device is essentially flow-less. To further elaborate the functionality of the OEIP together with amyloid-specific probes, we here present a multichannel OEIP that enables electronically controlled micron sized formation of plaquelike $A \beta$-aggregates with morphologies ranging from fine fibers, to bundles of fibers, and thick mesh-like fiber structures.

The OEIP is a versatile electrophoretic drug delivery device for precise, controlled nonconvection delivery of small ions or charged biomolecules to target solutions ${ }^{[13-15]}$. An encapsulated micrometer-scaled channel of a cation selective thin film membrane, produced by photolithography, is used as medium for the electrophoretic transport (Figure 1A). Either side of the channel is connected to a source and a target electrolyte and associated electrodes. By applying a driving voltage between the source and target electrodes, cations from the source electrolyte will migrate along the electric field through the channel and be released into the target electrolyte. To facilitate non-disruptive electron-to-ion conversion, Poly(3,4ethylenedioxythiophene):poly(styrenesulfonate) (PEDOT:PSS) electrodes are used, as those do not alter the $\mathrm{pH}$ or produce gases when operated within their capacity range ${ }^{[16]}$. The drug release rate into the target electrolyte is therefore controlled by the redox current applied to the electrodes. Local fibrous aggregation can be induced at the outlet of the OEIP channel by using a source electrolyte containing an aggregation-inducing species, such as protons that lowers the $\mathrm{pH}^{[17-19]}$, and a target electrolyte containing the actual peptide to be studied. The flat and thin OEIP devices is well suited for operation under microscope settings, and in 


\section{WILEY-VCH}

combination with fluorescent probes being specific to amyloid fibrils ${ }^{[20]}$, such as pFTAA $^{[12]}$, the aggregation of the peptides in the target electrolyte can be monitored and evaluated in real-time ${ }^{[11]}$. To increase the experimental throughput and to allow the same peptide solution to be used in multiple experiments, thus enabling more homogeneity between experiments, we designed a multichannel device with 6 parallel OEIP channels and utilized it in this study (Figure 1B).

We discovered the possibility of obtaining different aggregation conditions with the OEIP using identical $\mathrm{A} \beta 1-40$ solutions. $\mathrm{A} \beta 1-40(3.0 \mu \mathrm{M})$ and $\mathrm{HCl}(0.1 \mathrm{M})$ were added to the target and source, respectively, of 6 separate channels, and a delivery current, ranging from 100-400 nA (approximately 1-4 pmol $\mathrm{H}^{+} / \mathrm{s}$ ), was applied for 3 hours. To monitor the aggregation process pFTAA was used together with a fluorescent label (HiLyteFluor-555) covalently attached to a fraction of the $A \beta$ peptide. This allowed us to distinguish between peptide accumulation, detectable as an increase in HiLyteFluor-555 fluorescence, and fibril formation, detectable as an increase in pFTAA fluorescence. Images of the aggregation process were acquired with regular time intervals, see Figure 2. The presence of both pFTAA fluorescence, coded as green intensity, and HiLyteFluor-555 fluorescence, coded as red intensity, indicates that $A \beta$ peptide accumulated and formed fibril aggregates at all delivery rates. Notably the delivery rate could control the size and morphology of the A $\beta 1-40$ microscopic aggregates. The rate of the applied delivery current has a strong impact on the distribution of the fluorescence, with an increase in the overall size distribution with the applied current, showing that the rate of delivery can control the spread of aggregation. This effect can be related to an elevated concentration profile (established radially from the outlet) of the delivered protons with the delivery rate ${ }^{[11]}$. Also, low currents produce more uniform and more condensed fields of fluorescence, whereas high currents initially show more prominent ring effects that gradually convert into uniform fields of fluorescence over time. Aggregation starts already after 10 minutes and is detectable as weak fluorescence, which is followed by 


\section{WILEY-VCH}

continued aggregation and an increased fluorescence signal. In addition, at low currents we find a relatively higher HiLyteFluor-555 fluorescence signal as compared to the pFTAA fluorescence signal. This was particularly obvious at early time-points as expressed by the ratio of red/green fluorescence over time (Figure 2, last column of panels). At increased delivery currents the difference in HiLyteFluor-555 / pFTAA fluorescence ratio over time (i.e. between the first and last time point) is reduced, suggesting that higher currents are more optimal for producing amyloid-like aggregates, as opposed to predominantly forming an accumulation of $\mathrm{A} \beta$ (Figure 3).

At early time points the fluorescence is most intense along a truncated eclipse located around the outlet. This suggests that the aggregation starts here, and that this zone expresses the most favorable aggregation conditions. As the delivery rate of protons is increased, which lowers the $\mathrm{pH}$-profile around the outlet, this zone moves further away from the outlet, showing that the local $\mathrm{pH}$ is an important parameter for the aggregation kinetics ${ }^{[21]}$. Inside the eclipse the pFTAA fluorescence appears later and more diffuse, suggesting that the aggregation appears slower and that the morphology might differ. The pFTAA fluorescence is low outside the eclipse, while the HiLyteFluor-555 fluorescence spreads somewhat further away. This suggests that while $A \beta$ accumulates also outside the eclipse, the conditions do not favor aggregation into fibrillar structures. Experiments were also performed without pFTAA or labeled $\mathrm{A} \beta$, to control that the fluorphores did not interfere with each other (see Figure $\mathbf{S 1}$ in Supporting Information). A replicate of the experiment shown in Figure 2 is shown in Figure S3 in Supporting Information. For continuous studies a delivery rate of 300 nA was chosen, as this delivery rate resulted in a good mix of aggregation conditions.

Next we utilized scanning electron microscopy (SEM) to examine the final aggregates established after 3 hours of continuous application of a current followed by drying of the samples (Figure 4). The SEM study confirmed that formation of aggregates occur within the same area as where the fluorescence was observed in the fluorescence study. Detailed images 


\section{WILEY-VCH}

were taken at different spots of the aggregates that correspond to the eclipse, where aggregation was initially detected using fluorescence microscopy, and also inside and far outside of the eclipse. At the eclipse fine fibril structures were found, either separated or in the shape as bundles. Inside the eclipse, the aggregate formation was more disordered and less fibrillar to its characteristics. Here, the aggregate formations instead appeared to consist of densely packed structures. Outside the eclipse no aggregates were found whatsoever, only salt and other residues could be discerned. Thus, different morphologies for the aggregates was found in this SEM study, again suggesting that different aggregation conditions are present at varying distance from the outlet. It appears that the conditions at the eclipse were more favorable to form fine fibrillar structures. However, the more disordered aggregates inside the eclipse also showed pFTAA fluorescence, which indicates pFTAA-binding to fibrils. Finally, we examine the fibrillation of two different $A \beta$ peptides, $A \beta 1-40$ and $A \beta 1-42$, with the OEIP, as these different fragments of the $\mathrm{A} \beta$ peptide are known to differ in their aggregation kinetics, where $A \beta 1-42$ is regarded as the faster species to form aggregates as compared to $A \beta 1-40^{[22]}$. As seen in Figure 5, the overall aggregation process is similar for the two peptides, but with some notable differences. A $\beta 1-42$ showed a more granular appearance at the periphery of the aggregation zone displaying high pFTAA intensity, both early and late in the time-lapse. This suggests a faster aggregation for A $\beta 1-42$, where the accumulated peptide aggregates at early moments to form seeds from which further aggregation is promoted.

In conclusion, we report the application of a multi-channel OEIP device to control the formation of amyloid-like aggregates of the Alzheimer's disease associated amyloidogenic peptide $A \beta$. With the OEIP as a device to induce aggregation combined with amyloid fibrilspecific probes for detection, the kinetics, the micro-morphology, and the fibrillar nature of the formed aggregates can be followed in real time using fluorescent microscopy. The fibrillar nature, as well as distinct morphological difference of the aggregates depends on the 


\section{WILEY-VCH}

aggregation conditions (i.e. distance from the OEIP outlet), which is further confirmed in SEM studies. We foresee that the OEIP methodology can be implemented in multi array systems that can be utilized for studies of protein aggregation in confined spaces ${ }^{[23]}$ or together with cultured cells ${ }^{[24]}$ as well as for the development of screening platforms for assessment of molecules influencing the $A \beta$-aggregation process, and analysis of the specificity of fluorescent probes to amyloids ${ }^{[25]}$.

\section{Experimental Section}

OEIP manufacturing: OEIPs were produced according to previously reported methods ${ }^{[11,14,20]}$. PEDOT:PSS electrodes and cation-selective channels were patterned through photolithography and dry etching from sheets of Orgacon F-350 (PEDOT:PSS coated on polyethylene terephthalate, AGFA-Gevaert). A thin layer of Poly(methyl methacrylate) (Sigma-Aldrich, $\mathrm{M}_{\mathrm{w}} \sim 120000 \mathrm{~g} / \mathrm{mol}, 4 \mathrm{mg} / \mathrm{ml}$ in Diethyl carbonate) was spin coated (4000 $\mathrm{rpm}, 30 \mathrm{~s}$ ) on top of the substrate before patterning, to prevent photoresist delamination and enhance resolution. While the PEDOT:PSS electrodes were protected by a masking photoresist layer, the cation-selective channels were rendered electronically insulating by exposure to aqueous sodium hypochlorite ( 1 vol. \%) for 45 s. A $2 \mu \mathrm{m}$ thick SU8-layer (SU82002, Microchem) were deposited and patterned on top, to encapsulate the channels and to define openings for the liquid reservoirs. The reservoirs were produced by cutting circular openings into a $\sim 5 \mathrm{~mm}$ thick polydimethylsiloxane (PDMS, Dow Corning Sylgard 184) mold, and aligning the openings in the mold to the SU8 on the device. A $~ 1 \mathrm{~mm}$ thick PDMS-layer was used as a lid.

A $\beta$ aggregation using OEIPs: A similar setup as previously reported for amyloid-formation using OEIPs ${ }^{[11]}$ was used, but with modifications to accommodate 6 channels to be used in parallel. For all experiments, the source electrolyte contain $0.1 \mathrm{M} \mathrm{HCl}$ and the target electrolyte $0.1 \mu \mathrm{M}$ pFTAA and $3.0 \mu \mathrm{M} \mathrm{A} \beta(1-40$ or $1-42)$, of which $\sim 0.7 \%$ was HiLyteFluor-555-labeled A $\beta 1-40$. Fluorescence images were obtained with a Leica DM6000 


\section{WILEY-VCH}

B fluorescence microscope equipped with a SpectraCube camera in FISH-mode (Applied Spectral Imaging). $436 \mathrm{~nm}$ and $535 \mathrm{~nm}$ filter cubes were used to capture the fluorescence for the pFTAA and HiLyteFluor-555-probes, respectively, with $100 \mathrm{~ms}$ exposure time. For each point in the time-lapse, the pFTAA and HiLyteFluor-555 fluorescence was captured in series for each well before moving on to the next, producing a delay of $\sim 30 \mathrm{~s}$ between each well. After $3 \mathrm{~h}$, samples were dried by gently absorbing the target electrolyte with a paper tissue before the current was disconnected.

SEM study of formed aggregates: Dried samples were sputtered with $100 \AA$ A gold layer and viewed with a Gemini LEO 1550 microscope using an acceleration voltage of $5.00 \mathrm{kV}$.

\section{Supporting Information}

Supporting Information is available from the Wiley Online Library or from the author.

\section{Acknowledgements}

This work was supported by VINNOVA (2010-00507), the Swedish research council (20115804 and 621-2011-3517), the Advanced Functional Materials Center at Linköping University, and the Önnesjö foundation. K.P.R.N is financed by an ERC Starting Independent Researcher Grant (Project: MUMID) from the European Research Council. A generous gift from George and Astrid Olsson is acknowledged.

Received: ((will be filled in by the editorial staff))

Revised: ((will be filled in by the editorial staff)) Published online: ((will be filled in by the editorial staff))

[1] C. Ballard, S. Gauthier, A. Corbett, C. Brayne, D. Aarsland, E. Jones, The Lancet 19AD, 377, 1019.

[2] G. Irvine, O. El-Agnaf, Mol. Med. 2008, 14, 1.

[3] K. Islam, Y. C. Jang, R. Chand, S. K. Jha, H. H. Lee, Y. S. Kim, Journal of Nanoscience and Nanotechnology 2011, 11, 5657.

[4] T. P. J. Knowles, D. A. White, A. R. Abate, J. J. Agresti, S. I. A. Cohen, R. A. Sperling, E. J. De Genst, C. M. Dobson, D. A. Weitz, P Natl Acad Sci USA 2011, 108, 14746.

[5] Y. J. Choi, S. Chae, J. H. Kim, K. F. Barald, J. Y. Park, S.-H. Lee, Scientific Reports 2013, 3, 1921.

[6] M. Meier, J. Kennedy-Darling, S. H. Choi, E. M. Norstrom, S. S. Sisodia, R. F. Ismagilov, Angew. Chem. Int. Ed. 2009, 48, 1487. 


\section{WILEY-VCH}

[7] J. S. Lee, J. Ryu, C. B. Park, Anal. Chem. 2009, 81, 2751.

[8] J. S. Lee, C. B. Park, Biomaterials 2010, 31, 6789.

[9] J. S. Lee, E. Um, J.-K. Park, C. B. Park, Langmuir 2008, 24, 7068.

[10] V. Foderà, S. Pagliara, O. Otto, U. F. Keyser, A. M. Donald, J. Phys. Chem. Lett. 2012, 3, 2803.

[11] E. O. Gabrielsson, K. Tybrandt, P. Hammarstrom, M. Berggren, K. P. R. Nilsson, Small 2010, 6, 2153.

[12] A. Åslund, C. J. Sigurdson, T. Klingstedt, S. Grathwohl, T. Bolmont, D. L. Dickstein,

E. Glimsdal, S. Prokop, M. Lindgren, P. Konradsson, D. M. Holtzman, P. R. Hof, F.

L. Heppner, S. Gandy, M. Jucker, A. Aguzzi, P. Hammarström, K. P. R. Nilsson, ACS Chem. Biol. 2009, 4, 673.

[13] J. Isaksson, P. Kjaell, D. Nilsson, N. D. Robinson, M. Berggren, A. Richter-Dahlfors, Nature Materials 2007, 6, 673.

[14] K. Tybrandt, K. C. Larsson, S. Kurup, D. T. Simon, P. Kjall, J. Isaksson, M. Sandberg, E. W. H. Jager, A. Richter-Dahlfors, M. Berggren, Advanced Materials 2009, 21, 4442 .

[15] D. T. Simon, S. Kurup, K. C. Larsson, R. Hori, K. Tybrandt, M. Goiny, E. W. H. Jager, M. Berggren, B. Canlon, A. Richter-Dahlfors, Nat Mater 2009, 8, 742.

[16] P. G. Erlandsson, N. D. Robinson, Electrophoresis 2011, 32, 784.

[17] A. Aggeli, M. Bell, L. M. Carrick, C. W. G. Fishwick, R. Harding, P. J. Mawer, S. E. Radford, A. E. Strong, N. Boden, J Am Chem Soc 2003, 125, 9619.

[18] K. Giri, N. P. Bhattacharyya, S. Basak, Biophysical Journal 2007, 92, 293.

[19] M. Colaco, J. Park, H. Blanch, Biophysical Chemistry 2008, 136, 74.

[20] K. P. R. Nilsson, P. Hammarström, Advanced Materials 2008, 20, 2639.

[21] P. E. Fraser, J. T. Nguyen, W. K. Surewicz, D. A. Kirschner, Biophysical Journal 1991, 60, 1190.

[22] Y. Yan, C. Wang, Journal of Molecular Biology 2006, 364, 853.

[23] F. Librizzi, V. Foderà, V. Vetri, C. Lo Presti, M. Leone, Eur Biophys J 2007, 36, 711.

[24] C. J. Pike, D. Burdick, A. J. Walencewicz, C. G. Glabe, C. W. Cotman, Journal of Neuroscience 1993, 13, 1676.

[25] T. Klingstedt, A. Åslund, R. A. Simon, L. B. G. Johansson, J. J. Mason, S. Nyström, P. Hammarstrom, K. P. R. Nilsson, Organic and Biomolecular Chemistry 2011, 9 , 8356.

A

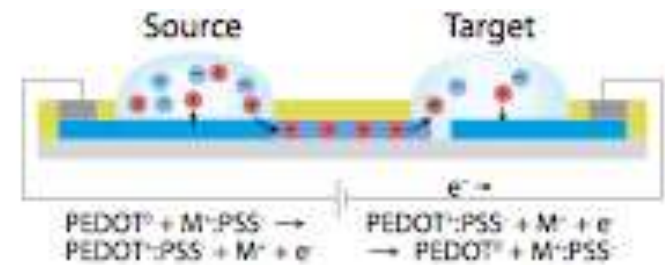

B
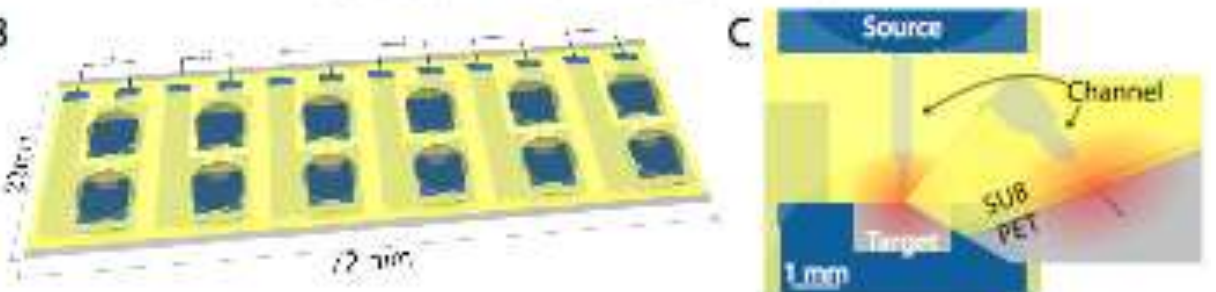

Figure 1. A) Side view of the electrophoretic cationic transport through an OEIP. B) Illustration of the 6 channel OEIP used in this work. The complete device measures $72 \times 23$ $\mathrm{mm}$ and fits on a standard glass slide. C) Close up of one device, showing the cation conductive channel connect source and target reservoirs. The channel tappers down to a width 


\section{WILEY-VCH}

of $50 \mu \mathrm{m}$ at the outlet. The outlet is defined by the edge of the SU8 encapsulation layer, from where delivered ions (red) radially diffuse out into the target electrolyte.

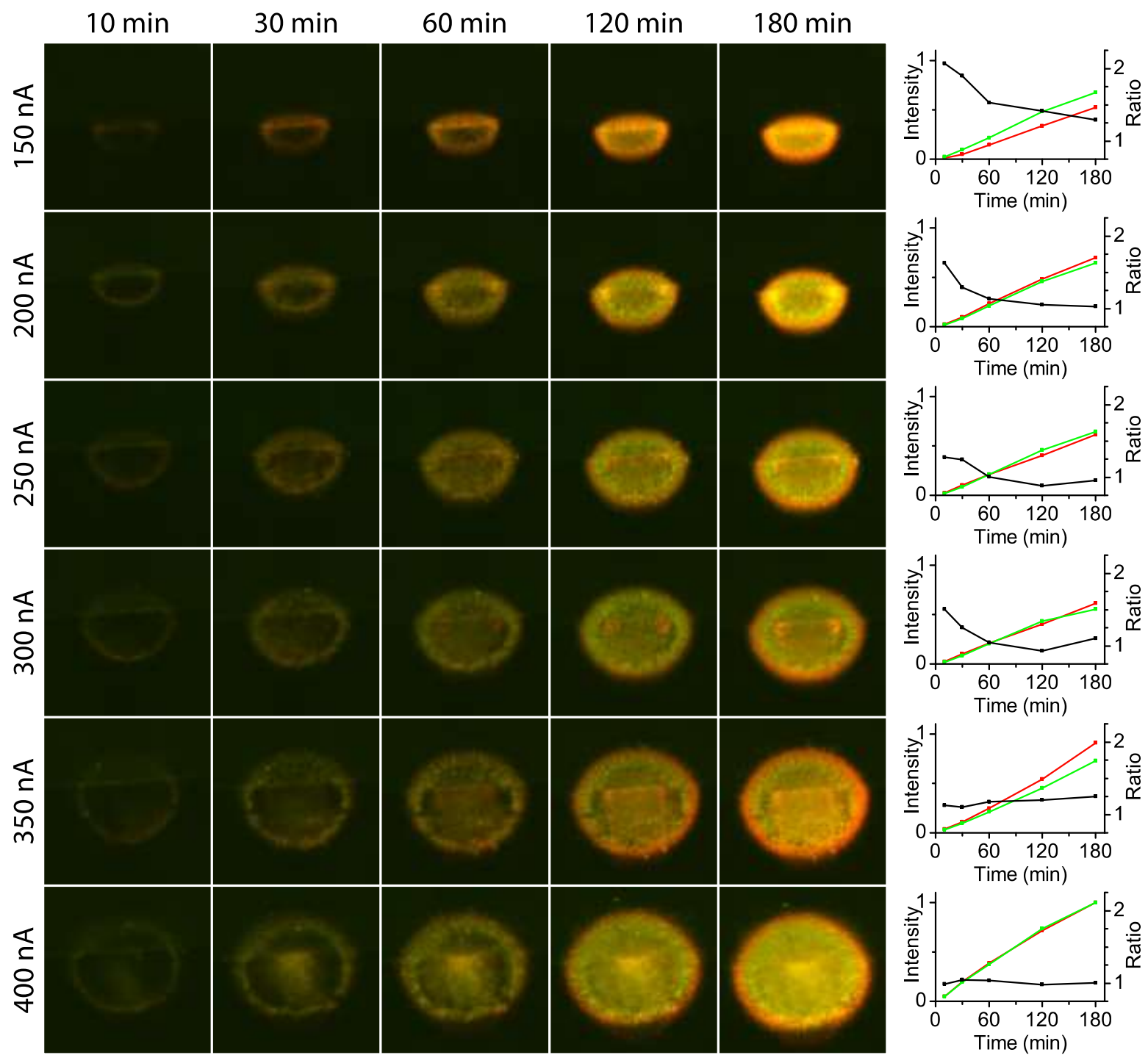

Figure 2. OEIP controlled formation of plaque like A $\beta$ structures. Fluorescent microscopy images of accumulation (HiLyteFluor-555, color coded red) and aggregation (pFTAA, color coded green) of A $\beta 1-40$ at 150-400 nA delivery current followed over 3 hours. The last panel in each row shows normalized integrated fluorescence intensities along a radial line passing through the aggregate versus time (see Figure S2 in Supporting Information).

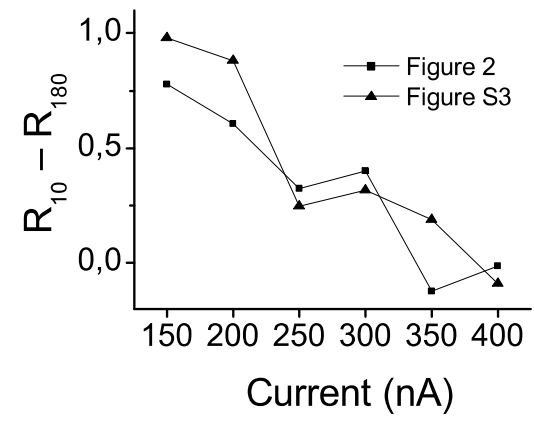

Figure 3. The difference ratio of red/green from early $(10 \mathrm{~min})$ and late $(180 \mathrm{~min})$ time points $\left(\mathrm{R}_{10}-\mathrm{R}_{180}\right)$ show a strong discrepancy of accumulation versus fibrillation at low currents. 


\section{WILEY-VCH}

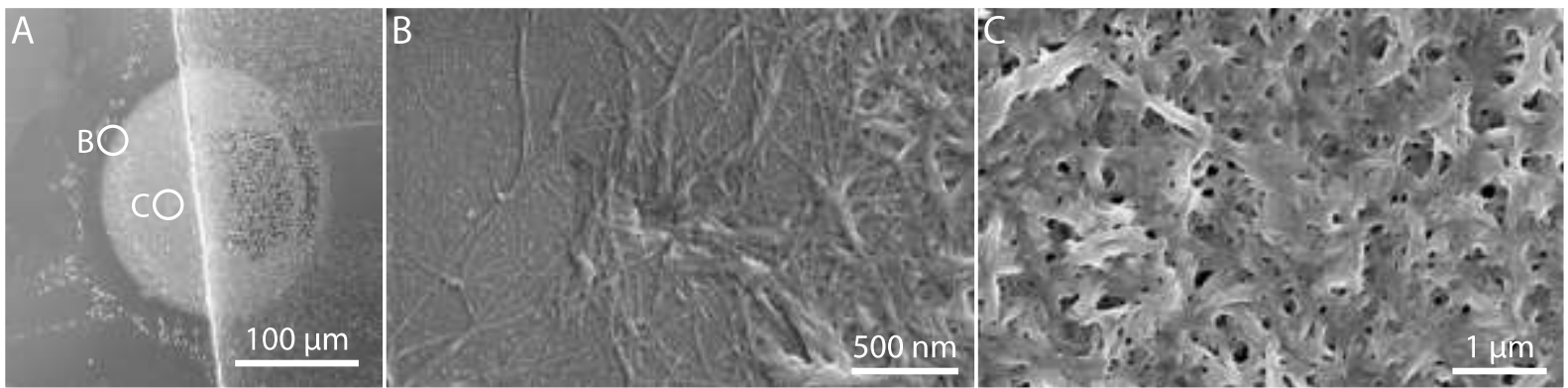

Figure 4. SEM images of dried A $\beta 1-40$ aggregates formed at $300 \mathrm{nA}$ after $3 \mathrm{~h}$. A) Overview of the circular aggregation zone. The image is rotated $90^{\circ}$ counter-clockwise compared to the representation in Figure 2. The line, going from top to bottom, is the edge of the encapsulation layer. The circles show the areas magnified in b) and c). B) Fine fibrils, around $20 \mathrm{~nm}$ in width, located at the perimeter of the eclipse (left side) while more densely packed fibril networks are accumulated towards the interior (right side). C) Densely packed aggregates located in the middle of the aggregation zone. The aggregates features are over $100 \mathrm{~nm}$ wide. 


\section{WILEY-VCH}

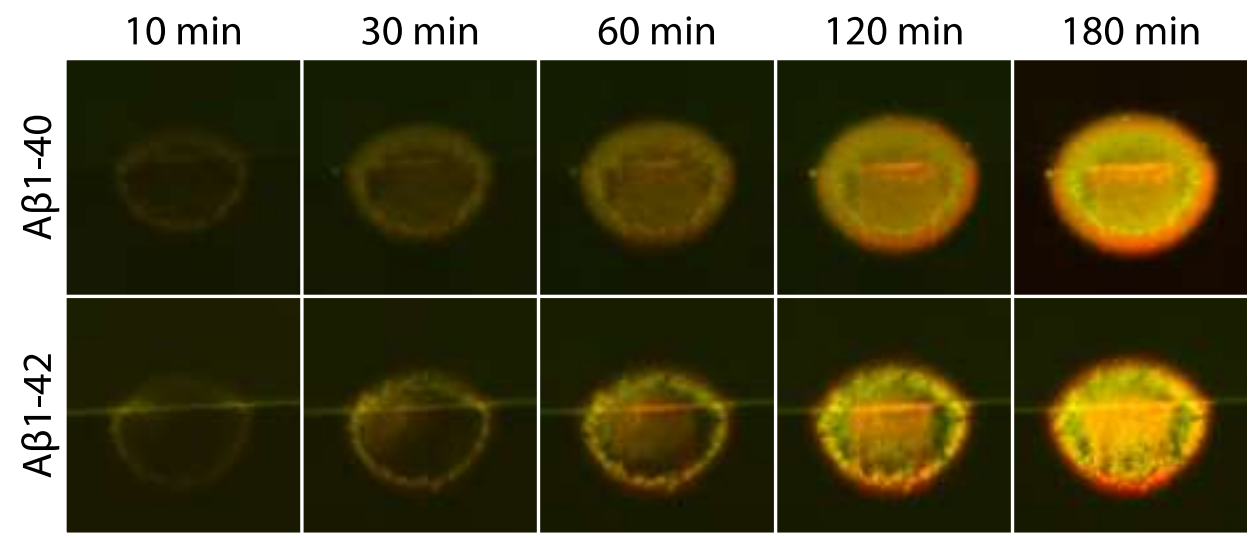

Figure 5. Aggregation of 3.0 $\mu \mathrm{M} A \beta 1-40$ and $\mathrm{A} \beta 1-42$ at $300 \mathrm{nA}$. A $\beta 1-42$ shows a more granular appearance of the formed microscopic aggregate. 


\section{WILEY-VCH}

An organic electronic device is used to to kinetically and spatially control Aß1-40 and A $\beta 1-42$ fibril formation. The formed aggregates show both nanometer-sized fibril structure and microscopic growth, thus mimicking senile plaques. Mechanistically, an initial accumulation of $A \beta 1-40$ is observed, likely driven by electrophoretic migration, which preceded nucleation of amyloid structures in the accumulated peptide cluster.

Amyloid, fibrils, Alzheimer's disease, bioelectronics, luminescent conjugated oligothiophene, optical probes

Erik O. Gabrielsson, Astrid Armgarth, Per Hammarström, K. Peter R. Nilsson ${ }^{*}$, and Magnus Berggren*

Spatiotemporal Control of Amyloid-Like A $\beta$ Plaque Formation Using a Multi-Channel Organic Electronic Device

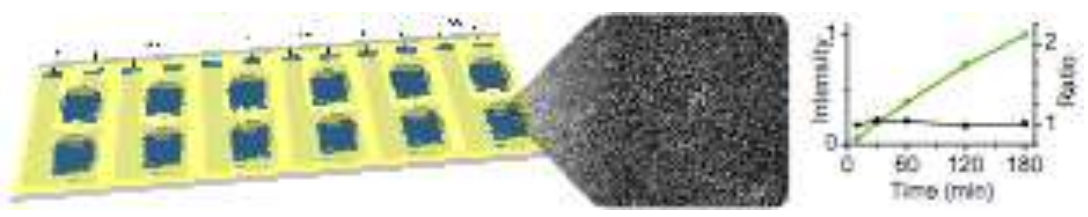




\section{WILEY-VCH}

Copyright WILEY-VCH Verlag GmbH \& Co. KGaA, 69469 Weinheim, Germany, 2013.

\section{Supporting Information}

Spatiotemporal Control of Amyloid-Like A $\beta$ Plaque Formation Using a Multi-Channel Organic Electronic Device

Erik O. Gabrielsson, Astrid Armgarth, Per Hammarström, K. Peter R. Nilsson*, and Magnus Berggren*

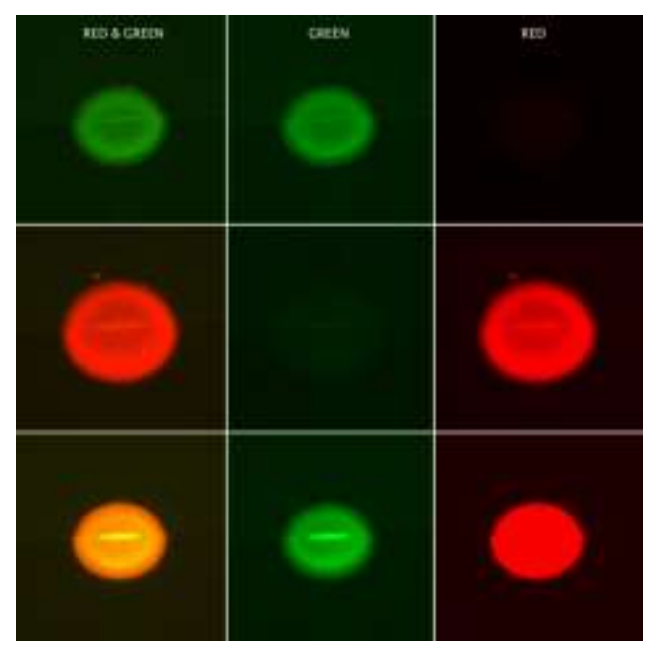

Figure S1. Spectral separation of fluorophores. (Top row) $0.1 \mu \mathrm{M}$ pFTAA and no HiLyteFluor-555-A $\beta 1-40$, (Middle row) no pFTAA and $0.7 \%$ HiLyteFluor-555-A $\beta 1-40$, (Bottom row) $0.1 \mu \mathrm{M}$ pFTAA and $0.7 \%$ HiLyteFluor-555-A $\beta 1-40$. Columns: filter-cubes red \& green combined, and green and red separately. 

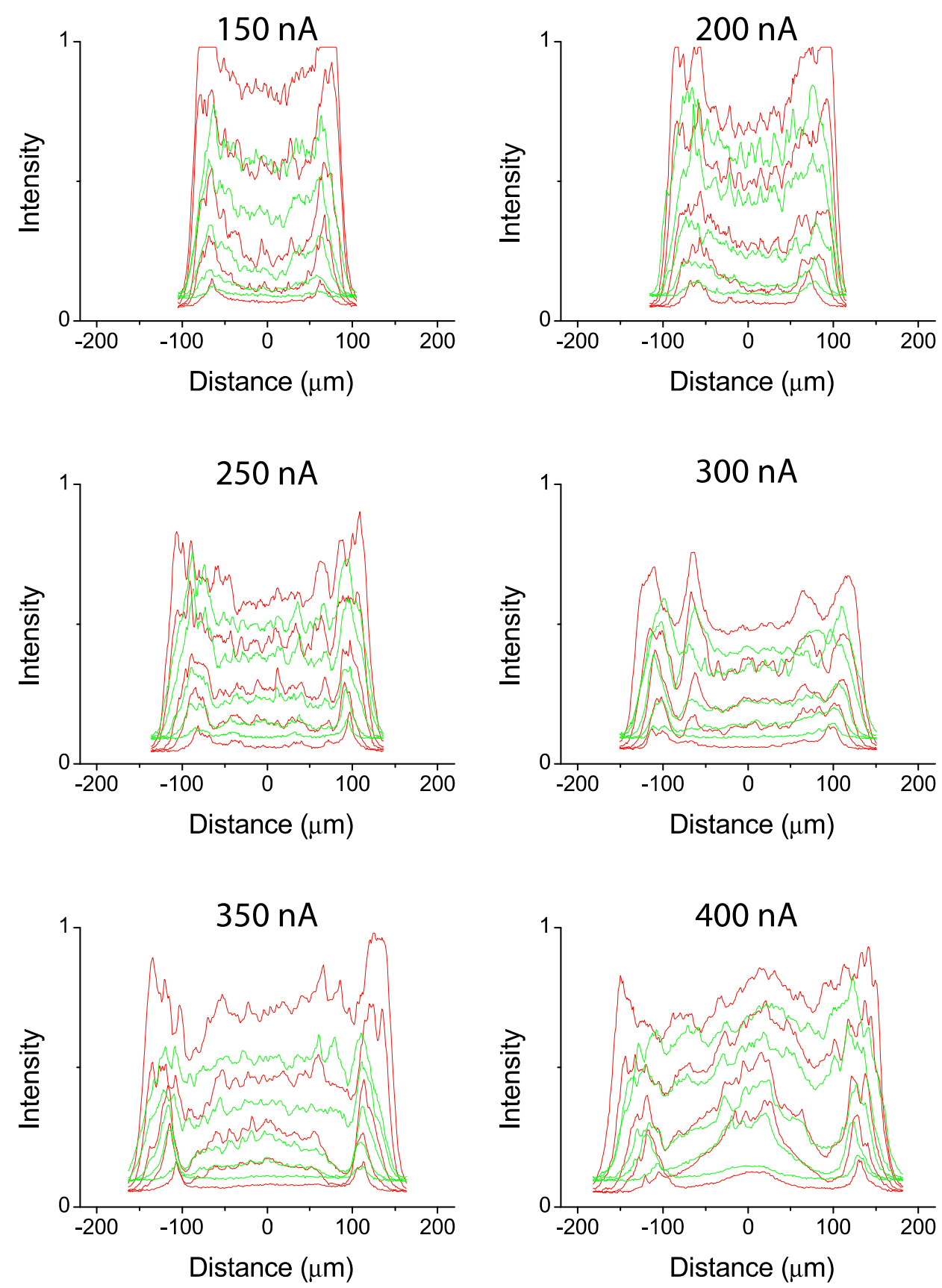

Figure S2. Fluorescence intensity profiles (red - HiLyteFluor-555, green - pFTAA) in plaque-like $A \beta 1-40$ structures grown at 150-400 nA over time (corresponding to Figure 2 in main article). 


\section{WILEY-VCH}

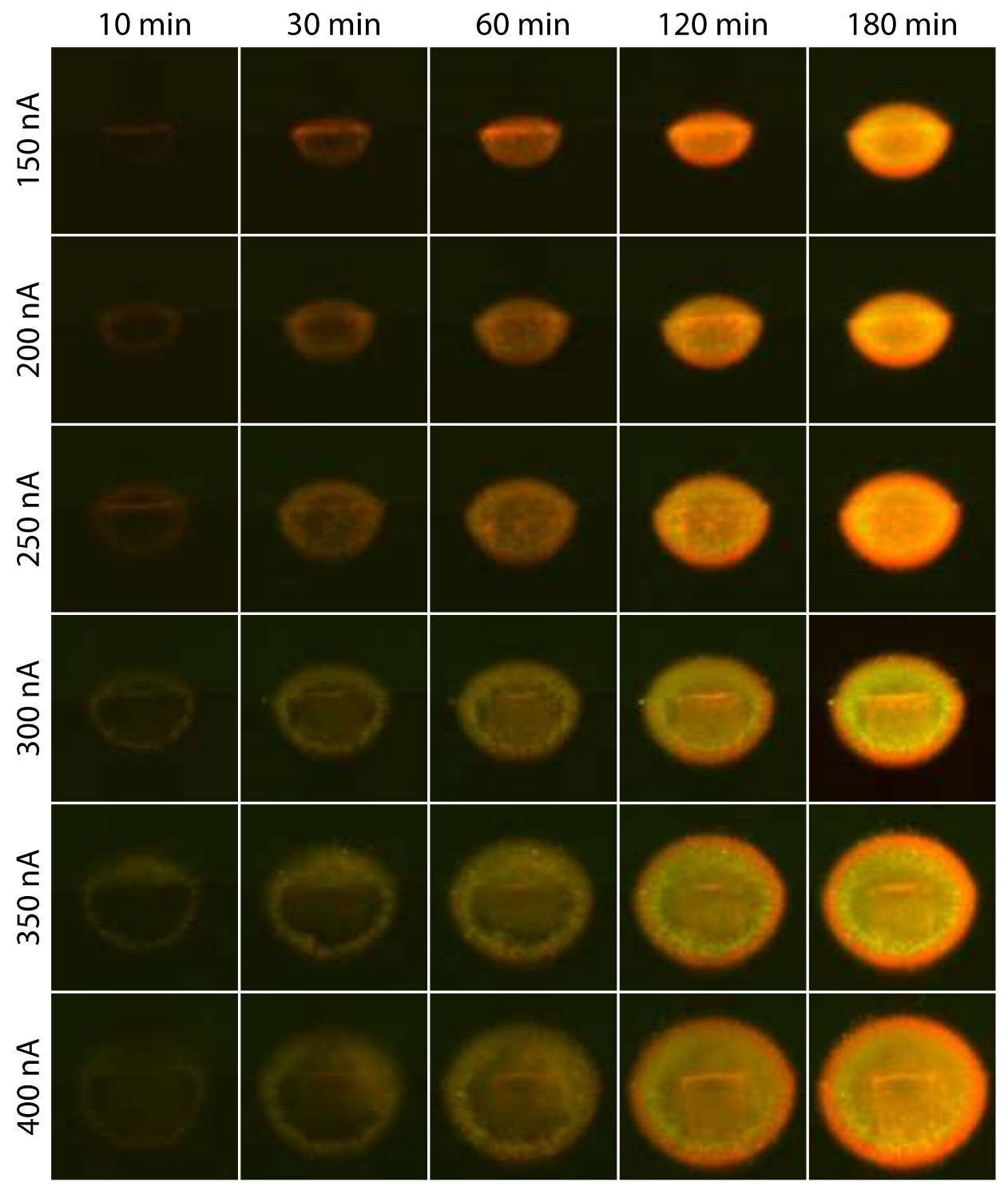

Figure S3. A replicate of the experiment shown in Figure 2. 